\title{
Estudio microbiológico del tracto respiratorio superior
}

\author{
STEPHANIE BRAUN J. ${ }^{1}$
}

\section{Microbiological procedures for diagnosis of upper respiratory tract infections}

Upper acute respiratory tract infections are very frequent diseases, specially in children. The bacteria involved in pharyngitis, acute otitis media and acute sinusitis, and the microbiological procedures for their study, are described.

Key words: Pharyngitis; Acute otitis media; Acute sinusitis; Microbiological procedures.

Las infecciones del tracto respiratorio superior, muy frecuentes como causa de consulta tanto a pediatras como a médicos internistas, incluyen entre otros síndromes la faringoamigdalitis aguda, la otitis media aguda y la sinusitis aguda.

\section{Faringoamigdalitis aguda}

La faringitis aguda en su gran mayoría obedecen a una etiología viral. Los virus involucrados más frecuentemente son rhinovirus, adenovirus, parainfluenza, y virus respiratorio sincicial, también virus Coxsackie, ECHO y herpes simplex. El virus de Epstein-Barr es un agente frecuente de faringitis y generalmente se manifiesta con las características clínicas de una mononucleosis infecciosa. Enfermedades sistémicas como citomegalovirus e influenza entre otras, pueden estar asociadas a faringitis aguda, como también la primoinfección del virus $\mathrm{VIH}^{1}$. La causa bacteriana más frecuente de faringoamigdalitis, y por lo tanto, susceptible de ser tratada con antimicrobianos, es Streptococcus pyogenes ( $\beta$ hemolítico grupo A), responsable de 15 a $30 \%$ de la faringoamigdalitis agudas en niños y de 5 a $10 \%$ en adultos. Aunque hay factores sugerentes de etiología viral como rinorrea abundante, disfonía, conjuntivitis, tos, diarrea, ausencia de fiebre y edad bajo 3 años, en muchos casos no se puede diferenciar mediante la semiología la etiología bacteriana y viral de una faringoamigdalitis y por lo tanto, deben utilizarse pruebas de laboratorio ${ }^{1,2}$. Los objetivos de tratar una faringoamigdalitis estreptocóccica son: prevención de enfermedad reumática, prevención de complicaciones supurativas (absceso periamigdalino, linfadenitis cervical, o mastoiditis), resolución de síntomas y signos, reducción de transmisión a contactos y minimizar los potenciales efectos adversos de los antimicrobianos $^{1}$.

\section{Cultivo faríngeo}

Es el método estándar de documentación de la presencia de $S$. pyogenes en la faringe, ya que una muestra única tiene 90 a $95 \%$ de sensibilidad para detectarlo ${ }^{1}$. Otro método que discutiremos más adelante es la detección rápida de antígeno, directamente de una muestra faríngea. El éxito de un cultivo o test rápido faríngeo depende de una buena toma de muestra.

Obtención de la muestra ${ }^{1-4}$.

- Utilice un bajalenguas para visualizar la faringe.

- Frote la tórula en ambas amígdalas y en la faringe posterior.

- Retire la tórula sin tocar la mucosa oral.

- Para transportar la tórula introdúzcala en un tubo estéril.

- Pueden haber falsos negativos con tratamiento antimicrobiano previo.

Laboratorio Hospital Militar del General Luis Felipe Brieba Arán. 


\section{Siembra}

Una vez tomada la muestra siembre en $1 / 6$ de una placa de agar sangre de cordero, haciendo cortes en profundidad de tal manera que se exprese la hemolisina O del Streptococcus. La primera lectura se hace a las $18-24 \mathrm{~h}$ buscando colonias $\beta$ hemolíticas. Si no se han desarrollado estas colonias, vuelva a incubar hasta $48 \mathrm{~h}$. La incubación anaerobia y el uso de medios selectivos pueden aumentar la proporción de cultivos positivos pero el impacto de éstos no justifican el aumento de costos y esfuerzos que involucra ${ }^{1}$. La incubación en $\mathrm{CO}_{2}$ favorece el desarrollo de otros patógenos faríngeos como Arcanobacterium haemolyticum ${ }^{1-5}$.

Para diferenciar S. pyogenes de otros estrepto$\operatorname{cocos} \beta$ hemolíticos se pueden usar distintos métodos.

Bacitracina: Esta prueba se basa en la susceptibilidad de $S$. pyogenes a la bacitracina. Se realiza mediante la colocación de un disco que contiene $0,04 \mu$ de bacitracina sobre la colonia aislada. Al día siguiente se determina la susceptibilidad a ella mediante la medición del halo de inhibición de crecimiento. Esta técnica tiene 5\% de falsos negativos, pero entre 10 y $20 \%$ de falsos positivos ya que hay otros Streptococcus (grupos C y $\mathrm{G})$, que también son susceptibles ${ }^{1-3,5,6}$.

Test de PYR. Es otro método muy utilizado y se basa en la actividad de la enzima pirronilodil aminopeptidasa producida por la bacteria. Streptococcus pyogenes es el único estreptococo que la sintetiza salvo algunos otros raramente aislados en clínica como $S$. iniae y $S$. porcinus. Una gran ventaja es su rapidez de ejecución y lectura $^{5}$. Esta prueba demostró tener $99 \%$ de sensibilidad y $100 \%$ de especificidad en un estudio de cepas chilenas ${ }^{6-7}$.

El método de PYR también es positivo en Enterococcus sp.

Los laboratorios que utilizan PYR o bacitracina deben informar: Hubo desarrollo presuntivo de Streptococcus pyogenes.

Ensayo inmunoenzimático. Una alternativa altamente específica es identificar los antígenos de carbohidrato de la pared grupo-específicos mediante serología, directamente sobre colonias aisladas. Hay numerosos kits comerciales disponibles para ello ${ }^{1,2,5}$. Este método sirve además para detectar Streptococcus del grupo C y G. Se debe diferenciar eso sí, las colonias pequeñas del grupo A que no son $S$. pyogenes sino Streptococcus del grupo anginosus. Los Streptococcus del grupo anginosus son responsables de abscesos en otras localizaciones pero se consideran comensales en la faringe ${ }^{5}$.
Es preferible que el informe de una muestra faríngea sea orientado con proyección clínica incluyendo los microorganismos que se buscaron, por ejemplo: No hubo desarrollo de Streptococcus $\beta$ hemolíticos A, C ni G.

Con respecto al estudio de susceptibilidad, en un servicio clínico donde se utiliza penicilina sódica no es necesario hacer estudio de susceptibilidad a ella ya que no se ha descrito resistencia a este fármaco. En muestras ambulatorias, con población que podría ser tratada con macrólidos, sí debe hacerse este estudio ya que se ha reportado 5,4 a $12 \%$ de resistencia a este grupo en Chile ${ }^{6-8}$.

Una desventaja del cultivo es que demora entre uno y dos días en entregar resultados y por eso se han desarrollado tests de detección rápida de antígenos.

\section{Tests rápidos de detección de antígenos}

El test rápido de detección de antígenos permite detectar al carbohidrato de la pared, directamente a partir de la tórula faríngea, con la ventaja de obtener resultados inmediatos. El uso de tests en servicio de urgencia ha permitido orientar el tratamiento más apropiado ${ }^{1}$.

La mayoría de los tests rápidos tienen excelente especificidad, > 95\%, comparados con cultivo, pero su sensibilidad es entre 80 y $90 \%$. Se recomienda entonces que un test negativo sea confirmado posteriormente con un cultivo ${ }^{1}$.

En otro estudio chileno se comparó un test rápido de detección de antígeno de EIA con cultivo faríngeo en 150 muestras, constatándose $28 \%$ de $S$. pyogenes y $2,6 \%$ de Streptococcus $\beta$ hemolíticos grupo $\mathrm{C}$ y G. La sensibilidad de EIA con respecto al cultivo fue de $90,5 \%$ y la especificidad de $99 \%$.

Los tests actuales están basados en técnicas de ELISA. Últimamente se han desarrollado el test con inmunoensayo óptico y sondas de ADN quimioluminiscentes. Los datos sugieren que son más sensibles que otros pero aún no están recomendados para uso rutinario ${ }^{1}$.

Salvo excepciones, no se recomienda hacer cultivo ni test rápido una vez completado el tratamiento antimicrobiano, ni a los contactos de pacientes con faringitis estreptocóccica ${ }^{1}$.

Otros Streptococcus que se encuentran con relativa frecuencia en muestras faríngeas son Streptococcus $\beta$ hemolíticos grupo C y G. Los Streptococcus grupo C y G se aíslan en 1,5\% de los cultivos faríngeos procesados en nuestro laboratorio. Estos han sido causantes de epidemias bien documentadas ${ }^{2,3}$. Los Streptococcus del grupo $\mathrm{C}$ y $\mathrm{G}$ expresan proteínas $\mathrm{M}$ similares con estructura a la de Streptococcus grupo A y las 
cepas virulentas del grupo $G$ expresan una peptidasa también similar al grupo $\mathrm{A}^{2,3}$.

Arcanobacterium haemolyticum ha sido reportado en Escandinavia, Sri Lanka y Reino Unido entre otros países, produciendo una faringoamigdalitis acompañada de rash máculopapular, en adolescentes y adultos jóvenes. La hemólisis aparece generalmente a las $48 \mathrm{~h}$ en agar sangre de cordero, y a las $24 \mathrm{~h}$ en agar sangre humano. Es un bacilo Gram positivo, catalasa negativo y se identifica por pruebas bioquímicas. También es característica una inhibición de la hemolisina de Staphylococcus aureus como prueba diagnóstica ${ }^{2,9}$. Es responsable de 0,5 a $2,5 \%$ de la faringitis bacterianas de adolescentes, en algunas series $^{2,9}$.

La angina de Vincent, producida por anaerobios mixtos, se puede diagnosticar mediante una tinción de Gram donde se aprecian fusobacterias y espirilos².

El resto de las causas bacterianas son de mucho menor frecuencia y su búsqueda debe ser orientada según las características clínicas y epidemiológicas del caso. Estas son Corynebacterium diphtheriae, Neisseria gonorrhoeae, Yersinia enterocolitica, Yersinia pestis y Franciscella tularensis ${ }^{2}$.

Hay microorganismos que son parte de la flora de la faringe y participan en la etiología de otras infecciones del tracto respiratorio pero no se consideran patógenos en faringoamigdalitis aguda en pacientes inmunocompetentes; es el caso de Haemophilus influenzae, Streptococcus pneumoniae, Moraxella catarrhalis y S. aureus.

En pacientes inmunocomprometidos, se deben informar el hallazgo de Candida sp, bacilos Gram negativos y $S$ aureus, ya que podrían tener un rol patógeno.

Para estudiar otras causas como N. gonorrhoeae, el diagnóstico debe ser dirigido por sospecha clínica y sembrar la muestra en agar Thayer Martin. A las colonias características se las estudia con tinción de Gram, test de oxidasa y fermentación de azúcares.

El estudio de $C$. diphtheriae también debe ser dirigido por sospecha clínica y se siembra en agar sangre de cordero, medio de Loeffler y agar telurito. Se diferencia de otros Corynebacterium por pruebas bioquímicas y se debe enviar a centro de referencia para pruebas de toxigenicidad ${ }^{2}$.

\section{Otitis media aguda y otitis externa}

\section{Otitis media aguda}

La otitis media aguda (OMA) es la inflamación del oído medio y se define como presencia de fluido en el oído medio acompañado de síntomas y signos de enfermedad aguda. Es muy frecuente en los primeros 3 años de vida. Aunque es menos frecuente en escolares y adolescentes, también puede producir fiebre, dolor y disminución de la audición en ellos y los adultos pueden sufrir sus secuelas.

Los microorganismos más frecuentemente encontrados en OMA son S. pneumoniae, $H$. influenzae tipo b y $H$. influenzae no tipificable, y M. catarrhalis. Otros microorganismos encontrados son $S$. pyogenes, $S$. aureus y virus como VRS, influenza enterovirus y rhinovirus ${ }^{10}$. Un estudio de OMA en niños chilenos, efectuado entre junio 1998 y junio de 1999, demostró una positividad de $82 \%$ de los cultivos, siendo $S$. pneumoniae aislado en $37 \%, \mathrm{H}$. influenzae en $24 \%, S$. pyogenes en $13 \%$, y $M$. catarrhalis en 2 casos. En este estudio se encontró virus en $13 \%$ de los casos y C. pneumoniae en 1 caso $^{11}$. En otro estudio efectuado en el Hospital Dr. Sótero Del Río, entre los años 2000 y 2001, $S$. pneumoniae fue aislado en $47,2 \%, H$. influenzae en $31,7 \%, S$. pyogenes en $6,5 \%, M$. catarrhalis en $5,7 \%, P$. aeruginosa en $1,2 \%$ y otros en $3,2 \%{ }^{12}$. En el primer estudio mencionado hubo $40 \%$ de cepas resistentes a penicilina (intermedia y resistentes) y $10 \%$ de Haemophilus productores de $\beta$ lactamasa, y en el segundo, el porcentaje de resistencia intermedia de $S$. pneumoniae a penicilina fue de $18 \%$ y de resistentes fue $4 \%$, habiendo además $8 \%$ de cepas de $H$ influenzae productoras de $\beta$ lactamasa ${ }^{11,12}$.

El diagnóstico de la OMA es generalmente clínico. La timpanocentesis sólo se efectúa con fines de investigación o en pacientes que no responden a la terapia instaurada.

La muestra de elección es el aspirado después de una timpanocentesis, pero si el tímpano ya se ha roto, se puede estudiar la secreción que fluye hacia el canal auditivo; se toma esta secreción con una tórula y se envía en medio de transporte de Stuart, de inmediato, al laboratorio para siembra en agar sangre de cordero, agar chocolate y caldo. Se incuba en $\mathrm{CO}_{2}$ durante $48 \mathrm{~h}$.

\section{Obtención de muestra ${ }^{4,11,12}$}

Método: Debe ser un procedimiento efectuado por especialista (otorrinolaringólogo). Bajo otomicroscopio se sigue el siguiente procedimiento:

Limpie el canal auditivo externo con solución antiséptica (alcohol al 70\%).

Aplique un anestésico local sobre el tímpano (solución de EMLA).

Efectúe timpanocentesis con una aguja adherida a un dispositivo especial.

Aspire el contenido. 
Siembre directamente en agar chocolate, agregue caldo y luego envíe al laboratorio. Se incuba en $\mathrm{CO}_{2}$ hasta $48 \mathrm{~h}$.

Se hace subcultivo en agar sangre de cordero a las $24 \mathrm{~h}$.

Las colonias aisladas se estudian mediante métodos rutinarios ${ }^{10,11}$.

\section{Otitis media con efusión}

La otitis media con efusión (OME) se caracteriza por la presencia de líquido en el oído medio sin signos de inflamación, observándose opacidad de la membrana timpánica, disminución de su movilidad y de la audición. La patogenia de esta entidad es multifactorial. En un estudio efectuado en Chile en 67 niños con OME en Chile, a los cuales se les practicó tímpanocentesis y se sembró el aspirado en un frasco de hemocultivo pediátrico Bact/Alert ${ }^{\circledR}$, se obtuvo bacterias patógenas en $37 \%$ de los cultivos. Estos fueron $S$. pneumoniae en 9\%, $H$. influenzae en $8 \%, M$. catarrhalis en $8 \%$ y $S$. aureus en $7 \%^{13}$.

\section{Otitis externa}

El canal auditivo externo tiene aproximadamente $2,5 \mathrm{~cm}$ de largo y es sinuoso. La flora externa del canal auditivo externo es similar a la piel de otras localizaciones, hay predominio de $S$. epidermidis, S. aureus, Corynebacterium sp y anaerobios como Propionibacterium acnes. Las células descamativas y la humedad retenida lo hacen especialmente susceptible a bacterias hidrofílicas como $P$. aeruginosa.

La otitis externa aguda o enfermedad del nadador ocurre especialmente en pacientes que están expuestos al agua, El pabellón auricular se torna edematoso, eritematoso y doloroso. Los patógenos más frecuentemente encontrados son $P$. aeruginosa, S. aureus y Streptococcus $\mathrm{sp}^{10,14}$.

\section{Obtención de muestra}

Limpie el canal auditivo externo con abundante solución salina fisiológica estéril. Tome la muestra con un tórula sobre las lesiones presentes ${ }^{3}$.

Siembre en agar sangre y Mac Conkey y caldo.

En la otitis externa invasora o "maligna", la infección invade los tejidos adyacentes pudiendo llegar a ser muy severa; la diabetes mellitus es un factor predisponente. En este caso se debe obtener un cultivo profundo de tejido para su cultivo ${ }^{10,14}$.

\section{Sinusitis aguda}

La sinusitis es una infección de una o más cavidades paranasales. Puede ser clasificada en varias categorías según si es adquirida en la comunidad o nosocomial, según el estado inmunológico del paciente o según sus agentes etiológicos (virus, bacterias u hongos).

La sinusitis maxilar aguda en pacientes inmunocompetentes, que la adquieren en la comunidad, es la más común.

Los microorganismos más frecuentemente involucrados son virus, virus asociados con bacterias, o bacterias. Los hongos que son mucho menos frecuentes, producen generalmente cuadros no invasores en pacientes inmunocompetentes.

Los microorganismos más frecuentes son $S$. pneumoniae, $H$. influenzae y en menor proporción, $M$. catarrhalis ${ }^{10,15}$.

En estudios chilenos se ha encontrado $S$. pneumoniae en 23 a 40\%, Haemophilus en 40\%, Streptococcus $\beta$ hemolíticos en 3 a $17 \%$, y $M$. catarrhalis en $10 \%$ de los $\operatorname{casos}^{16,17}$. En otro estudio efectuado en 39 pacientes se aisló anaerobios en $21,4 \%$ de ellos, de los cuales $10,3 \%$ estaban asociados a bacterias aerobias ${ }^{18}$.

\section{¿Es necesario hacer diagnóstico bacteriológico en una sinusitis aguda?}

En la mayoría de los casos no, ya que el tratamiento se realiza en forma empírica, basado en los estudios de investigación.

Las indicaciones de tomar una muestra para cultivo son: paciente séptico o inmunocomprometido, infección nosocomial generalmente en pacientes intubados, si hay mala respuesta a la antibioterapia y, en presencia de complicaciones como meningitis, absceso cerebral, celulitis periorbitaria y osteomielitis ${ }^{10,15,21}$.

\section{Sinusitis maxilar aguda nosocomial}

Está asociada generalmente a intubación nasoentérica o naso-traqueal en pacientes sometidos a ventilación mecánica.

En un estudio se encontró cultivos polimicrobianos en $82,1 \%$ de los casos, $P$. aeruginosa y Enterobacteriaceas, y las cocáceas Gram positivas como $S$. aureus, aisladas con mayor frecuencia; además se aislaron anaerobios y levaduras. En otro estudio de 20 pacientes, 55\% fueron polimicrobianos, bacilos Gram negativos se aislaron en $60 \%$ de los casos, especialmente Acinetobacter baumannii, y entre las cocáceas Gram positivas, S aureus y Enterococcus, fueron frecuentes. En este estudio se comparó cultivo de tejido obtenido bajo visualización endoscópica con la muestra tomada por punción sinusal encontrándose $60 \%$ de correlación entre ellos. 
El diagnóstico etiológico se hace mediante punción aspirativa del seno maxilar o mediante cultivo de tejido o secreción obtenida mediante endoscopia ${ }^{19,20}$.

\section{Sinusitis maxilar aguda en paciente inmunocomprometido}

En pacientes sometidos a tratamiento por enfermedades neoplásicas, en trasplantes de precursores hematopoyéticos y de órganos sólidos, en diabetes mellitus, además de los mismos agentes bacterianos encontrados en la sinusitis aguda se agregan los patógenos fúngicos invasores como Mucorales y Aspergillus ${ }^{10,21}$. En pacientes con SIDA, los más frecuentes son los mismos que en la población general, pero se agregan otros como $S$. aureus, $P$. aeruginosa y Aspergillus sp, entre otros $^{21}$. En un estudio chileno de sinusitis maxilar en 12 pacientes infectados por VIH, se encontró $S$. pneumoniae en 4, $H$. influenzae en $3, M$. catarrhalis en 2 y $P$. aeruginosa en $1^{22}$.

La punción del seno maxilar es el estándar para obtener una buena muestra en una sinusitis, pero este procedimiento es doloroso y puede tener complicaciones. Se han hecho estudios comparando muestras de secreción obtenidas bajo visualización directa del meato sinusal mediante un endoscopio rígido, con muestras obtenidas por punción. Talbot estudió 46 pacientes con ambas técnicas, y observó 85,6\% de sensibilidad y $90 \%$ de especificidad si consideraba solamente $S$. pneumoniae, Haemophilus sp y $M$. catarrhalis, obteniendo eso sí un mayor aislamiento de $S$. aureus que puede ser parte de la flora del meato ${ }^{23-26}$. Vogan obtuvo en 16 pacientes $90 \%$ de correlación.

\section{Toma de muestra $a^{4,15,17}$}

Debe ser realizada por un especialista (otorrinolaringólogo).

\section{Cultivo obtenido por punción maxilar:}

Se desinfecta la parte anterior de la fosa nasal y el área debajo del cornete inferior (sitio de punción).

Se anestesia la zona del procedimiento.

Se punciona la pared medial del seno.

Se aspira el contenido en una jeringa y se agrega 1 a $2 \mathrm{ml}$ de solución salina si es necesario.

Se envía al laboratorio de inmediato y una porción en medio de transporte para anaerobios.

\section{Cultivo a través de endoscopio}

Se anestesia localmente la cavidad nasal.

Mediante un endoscopio rígido se inspecciona el meato medio para observar secreciones.
Se toma la muestra mediante una tórula de alginato. Se coloca en medio de Stuart y en medio de transporte para anaerobios.

Siembra: algunos utilizan método cuantitativo.

Se siembra en agar sangre de cordero, agar chocolate y caldo TSB, incubándose durante 48 $\mathrm{h}$ en $\mathrm{CO}_{2}$. Además se siembra en anaerobiosis: agar sangre para anaerobios (hemina), agar kanamicina y tioglicolato.

Las muestras nasales tienen poca correlación con las obtenidas por punción sinusal. Axelson comparó cultivos nasales con punción maxilar y obtuvo sólo $65 \%$ de correlación entre ellos. Wald comparó muestras nasofaríngeas con punción sinusal (47 casos) y de 17 pacientes con predominio de un patógeno en nasofarinx, 4 crecieron en el cultivo de la punción sinusal. Evans encontró que en $44 \%$ de los cultivos aislados en punción no se encontró la misma bacteria en el cultivo nasal ${ }^{27-29}$.

\section{Conclusiones}

En la faringoamigadalitis aguda es recomendable buscar la presencia de $S$. pyogenes en la faringe mediante un test rápido o mediante cultivo, ya que clínicamente pueden ser indistinguibles una causa viral de una bacteriana.

En OMA y en sinusitis, en la gran mayoría de los casos no es necesario hacer un diagnóstico microbiológico ya que se tratan en forma empírica según resultados de estudios de investigación. Las bacterias más frecuentes en ambas patologías son las mismas, $S$ pneumoniae, $H$. influenzae $\mathrm{y}$ en menor porcentaje, $M$. catarrhalis.

\section{Resumen}

Las infecciones del tracto respiratorio superior son muy comunes, particularmente en niños. Se describen las bacterias que producen faringitis, otitis media aguda y sinusitis aguda y los procedimientos microbiológicos empleados en su identificación.

\section{Bibliografía}

1.- Bisno A, Gerber, Guerree MA, Gwaltney JM, Kaplan EL, Schwartz RH. Diagnosis and management of group A streptococcal pharyngitis: a practice guideline. Clin Infect Dis 1997; 25: 574-83.

2.- Gwaltney JM, Bisno AL, Pharyngitis, En Mandell, Douglas and Bennett's Principle and Practice of 
Infectious Diseases, Mandell G L, Bennett J E and Dolin R, eds, 5th edition, 2000. Churchill Livingstone Philadelphia, pp: 656-62.

3.- Johnson CC, Tunkel AR. Viridans Streptococci and groups $\mathrm{C}$ and G Streptococci. En Mandell, Douglas and Bennett's Principle and Practice of Infectious Diseases, Mandell GL, Bennett JE and Dolin R, eds, 5th edition, 2000. Churchill Livingstone Philadelphia, pp 2167-83.

4.- Miller J M. Throat and nasopharyngeal specimens. En Miller J M, A Guide to Specimen Management in Clinical Microbiology. American Society of Microbiology Washington DC 1996, pp 31-2.

5.- Ruoff K L, Whiley R A, Beighton D. Streptococcus. En: Manual of Clinical Microbiology. PR Murray, EJ Baron, MA Pfaller, FC Tenover, RH Yolken, eds, 7th edition, 1999. American Society of Microbiology Press Washington D C, pp 283-96.

6.- Giglio M S, Ulloa M T, Robles M, Garay B, Rojas P, Pinto M E. Uso de bacitracina, 1-piroglutamil amilaridasa y aglutinación en el diagnóstico de Streptococcus $\beta$-hemolítico. Rev Chil Infect 1997; 1115: 39-44.

7.- Ulloa M T, Becker L, Robles M, Giglio M, Camponovo R. Comparación de StrepA y cultivo tradicional en el diagnóstico de Streptococcus $\beta$ hemolítico grupo A. XXI Congreso Chileno de Microbiología, Valdivia 1999, Asociación Chilena de Microbiología.

8.- Giglio M S, Porte L, Herve B, Ulloa M T. Susceptibilidad in vitro de patógenos respiratorios a levofloxacina y otros antimicrobianos. Rev Chil Infect 2000; 17: 18-24.

9.- Linder R. Rhodococcus equi and Arcanobacterium haemolyticum: two "coryneform" bacteria increasingly recognized as agents of human infection. Emerg Infect Dis 1997; 3: pp 2, download article.

10.- Klein J O. Otitis externa, Otitis media and mastoiditis, Gwaltney J.M., Sinusitis, p 676-686, 2000 In Mandell, Douglas and Bennett's Principle and Practice of Infectious Diseases, Mandell GL, Bennett JE and Dolin $\mathrm{R}$, eds, $5^{\text {th }}$ edition, 2000 Churchill Livingstone Philadelphia, pp 669-76.

11.- Rosenblut A, Santolaya M E, González P et al. Bacterial and viral etiology of acute otitis media in Chilean children. Pediatr Infect Dis J 2001; 20: 501-7.

12.- González P A. Comunicación personal.

13.- Beltrán C, Fonseca X, Guzmán A M, Arredondo M, González C, Cohen M. Otitis media con efusión: estudio bacteriológico optimizado para microorganismos aeróbicos Libro de Resúmenes, LVIII Congreso Chileno Otorrinolaringología y Cirugía de Cabeza y Cuello.

14.- Durand M, Joseph M, Sulliva Baker A. Infections of the upper respiratory tract. En Harrinson's Principles of Internal Medicine, Fauci A. et al. eds, 14 th edition,1998, The Mc Graw-Hill Companies, U.S.A, pp:179-84.

15.- Gwaltney J M. Acute community-acquired sinusitis. Clin Infect Dis 1996; 23: 1209-25.

16.- Correa A, Cabeza Y, Corssen C. Sinusitis maxilar aguda. Rev Otorrinolaringol Cir Cabeza Cuello 1994; 54 : 73-5.

17.- Siri M T, Fonseca X, Prado V, Díaz M C. Microbiología de la sinusitis aguda. Rev Chil Infect 1990; 7: 258.

18.- Constanzo C, Contreras J M, Bustos L, Paredes L. Anaerobios en sinusitis maxilar aguda. Rev Otorrinolaringol Cir Cabeza Cuello 1993; 53: 35-9.

19.- George D L, Falk P S, Meduri G U et al Nosocomial sinusitis in patients in the medical intensive care unit: a prospective epidemiological study. Clin Infect Dis 1998; 27: 463-70.

20.- Casiano R, Cohn S, Villasuso E et al. Comparison of antral tap with endoscopically directed nasal culture. Laryngoscope 2001; 111: 1333- 7.

21.- Pankey G A, Gross C W, Mendelsohn M G. Contemporary diagnosis and managemente of sinusitis, 1997, Handbooks in Healthcare Co., Pennsylvania.

22.- Noriega L M, Rosenblut A, Pérez J. Sinusitis en pacientes VIH (+). Rev Otorrinolaringol Cir Cabeza Cuello 1996; 56: 15-7.

23.- Talbot G H, Kennedy D W, Scheld M, Granito K. Rigid nasal endoscopy versus sinus puncture and aspiration for microbiologic documentation of acute bacterial maxillary sinusitis. Clin Infect Dis 2001; 33 : 1668-75.

24.- Vogan J C, Bolger W E, Keyes A S. Endoscopically guided sinonasal cultures: a direct comparison with maxillary sinus aspirate cultures. Otolaryngol Head Neck Surg 2000; 122: 370-3.

25.- Klossek J M, Dubreuil L, Richet H, Richet B, Sedallian A, Beutter P. Bacteriology of the adult middle meatus. J Laryngol Otol 1996; 110: 847-9.

26.- Gordts F, Halewyck S, Pierarad D, Kaufman L, Clement P A R. Microbiology of the middle meatus: a comparison between normal adults and children. J Laryngol Otol 2000; 114: 184-8.

27.- Axelson A, Brorson J E. The correlation between bacteriological findings in the nose and maxillary sinus in acute maxillary sinusitis. Laryngoscope 1973; 83: 2003-11.

28.- Wald E R, Milmoe G J, Bowen A, Ledesma-Medina J, Salamon N, Bluestone C D, Acute maxillary sinusitis in children. N Eng J Med 1981; 304: 749-54.

29.- Evans F O, Sydnor J B, Moore W E C et al. Sinusitis of the maxillary antrum. N Eng J Med 1975; 293: 735-9.

Correspondencia a:

Stephanie Braun Jones

E-mail: stbraun@123mail.cl 